\title{
Adherence in CABG Patients: An Empirical Test of a Health Behavior Model
}

\author{
Fariba Zarani ${ }^{*}$, Gholamreza Sarami², Saeed Sadeghian ${ }^{3}$ \\ ${ }^{1}$ Department of Education \& Psychology, University of Shahid Beheshti, Tehran, Iran \\ ${ }^{2}$ University of Kharazmi, Tehran, Iran \\ ${ }^{3}$ Tehran University of Medical Sciences, Tehran, Iran \\ Email: fzarani@yahoo.com
}

Received 11 December 2013; revised 10 January 2014; accepted 9 February 2014

Copyright (C) 2014 by authors and Scientific Research Publishing Inc.

This work is licensed under the Creative Commons Attribution International License (CC BY). http://creativecommons.org/licenses/by/4.0/

(c) (i) Open Access

\section{Abstract}

To benefit from a Coronary Artery Bypass Graft Surgery (CABG), patients must adhere to their therapeutic regimen. To test the extent of which the Information-Motivation-Behavioral Skills (IMB) model constructs explain the variability in adherence behaviors in CABG patients, and also to examine the relationship between the IMB model constructs, CABG patients $(N=152)$ were randomly assigned to either an intervention group, or a standard care control group. They completed pretest measures, and were reassessed later. Although perfect replication of the IMB model's predicted pathways was not achieved in this study, support for several of these pathways was demonstrated. The estimated model for adherence-demonstrated good fit of the data. Motivation was a significant predictor of adherence behavior in CABG patients. These findings suggest that IMB model-based intervention that improves motivation can enhance adherence. Therefore, CABG patients' education programs should mainly target motivation in order to affect adherence behavior.

\section{Keywords}

Adherence; Information-Motivation-Behavioral Skills Model; CABG Patients; Health Behavior Model

\section{Introduction}

Secondary prevention is imperative for patients undergoing invasive coronary procedures such as CABG [1]. It is of great importance that patients undergoing CABG maintain their compliance to lifestyle changes and adhe-

"Corresponding author. 
rence to drug treatment in order to benefit in long term. Thus the first step in helping patients with cardiovascular disease is determining the factors that could influence the patients' adherence. There is a plenty of evidence to support that provision of information [2] [3], focusing on personal motivation to change unhealthy life habits [4] [5], autonomous regulation and self-efficacy [6] [7], and implementation of behavioral techniques [5] could enhance adherence in CHD patients. Therefore, patients should receive proper information, education and support to be able to change their lifestyles, and to maintain these changes, and comply with therapeutic interventions [8]. Despite the strong body of evidence that describes optimum medical care and best practice [9] [10], however, evidence suggests that there are significant gaps in the implementation of secondary prevention that patients receive. So far, clinical interventions to enhance adherence have not had sufficient theoretical basis. Researchers often adopt a "cafeteria-style" intervention design in which constructs are selected from a range of theories and models and then mixed together [11]. Examining a construct out of the context of a full model reduces the validity and utility of the construct, since different theories could often be based on contrasting assumptions. Thus, high-quality experimental tests of theories are much needed in clinical populations [12].

Application of interventions with demonstrated effectiveness will have useful implications for clinical practice. Theory-driven interventions and studies of single theory clinical trials allow unambiguous examination of the entire theoretical models, and their efficiency to identify the factors that predict sustained behavioral changes [7] [13].

The Information-Motivation-Behavioral Skills (IMB) model of health behavior is a theoretical model developed in 1992 to predict HIV-preventive behavior; however, its concepts could be broadly applied to predict positive health behavior changes in a variety of contexts [14]. The IMB model of health behavior change posits that performing a health promotion behavior is a function of the extent to which someone is 1) well informed about the behavior, 2) motivated to perform the behavior (e.g., has positive personal beliefs and attitudes towards the behavior or outcome, and social support for the behavior), 3) equipped with the required skills to execute the behavior, and 4) confident in his ability to do so across various situations [15] [16].

Essentially, one who is well informed and motivated is more likely to develop and apply the skills necessary to enact the required health promotion behavior and to ultimately reap greater health benefits [15] [16].

The model's constructs and the relationship among them have been well-supported across various populations and with different health promotion behaviors [15] [16], however, little research has been done in patients with heart disease.

The recently developed Information-Motivation-Behavioral skills (IMB) model [16] [17] has integrated elements into the model to construct a conceptually based, generalizable, and parsimonious model that could explain complex health behaviors [4], and its concepts could be broadly applied to predict positive health behavior changes in a range of contexts [14]. Thus adherence-related information, motivation, and behavioral skills are the fundamental determinants of adherence behavior.

In their previous research, the authors evaluated primary outcome of IMB intervention [18], and examined the effectiveness of intervention [19] in CABG patients.

The IMB model specifies the structural relationship among the information, motivation, and behavioral skills constructs with adherence. Consistent with this conceptual model, adherence-related information and motivation were significantly correlated with adherence-related behavioral skills, and behavioral skills were significantly correlated with adherence behavior [20].

The IMB model asserts that specific constructs of the model and specific relationships among them will predict different health behaviors in different populations. (See Fisher \& Fisher, 2000 for a review of this literature).

Through a series of path analysis, we sought to evaluate the information (adherence behaviors related knowledge), motivation (personal and social), and behavioral skills (adherence behaviors related skills) components of the IMB model with respect to self-care behavior.

By systematically testing elements of health behavior change theories, investigators can verify the accuracy of predictions, and develop a better understanding of their practical value [13]. Thus testing theoretical models across population are needed to specify their scope and utility. The present study extended previous research in an important way by testing the utility of the IMB model in designing interventions to promote adherence in patients with cardiovascular disease.

This paper entails some secondary analysis from a previous randomized study [18]. The goals of current research were 1) to test the extent to which IMB model constructs explain the variability in adherence behaviors in CABG patients, and 2) to examine the relationship between the IMB model constructs in order to introduce 
modifications to the IMB model and the IMB model-based intervention for heart patients. The novelty of this paper lies in developing tailored intervention for CABG patients on the basis of IMB model elements.

\section{Methods}

\subsection{Participants}

One hundred fifty-two Coronary Artery Bypass Surgery (CABG) patients participated in a randomized controlled study. A detailed description of the methods, including patient selection and the primary findings has been reported previously [18]. The present study was conducted from April to August 2007, and involved patients that had been diagnosed through routine angiography. These patients had been admitted for elective Coronary Artery Bypass Grafting (CABG) at the cardiothoracic surgery unit of Tehran Heart Center in Iran, and were considered potentially eligible for the study. Inclusion criteria were 1) age range between 40 and 65 years old, 2) access to medical care, 3) diagnosis of heart problem for more than a year, 4) ability to read and write, 5) absence of visual/hearing impairments, 6) absence of co-morbid conditions (i.e., other severe somatic diseases, psychosis). A total of 180 eligible participants were randomly assigned to the intervention group $(n=90)$ or standard care control group $(n=90)$. The patients in both groups completed pretest measures. Participants who did not return a month later for posttest re-assessment $(n=28)$ were excluded from the final analyses. Fifteen of the 28 patients later cancelled their appointments. Twelve of the 28 patients developed serious health problems (i.e., pulmonary edema) and could not continue to participate, and one of the patients passed away. The final sample consisted of 152 patients (124 males, 28 females; 149 married, 3 single; age range 40 - 60 years old, $\mathrm{M}=$ 53.15, $\mathrm{SD}=11.87)$.

\subsection{Measures}

\subsubsection{Demographic Measure}

Participants answered questions regarding their gender, age, educational level and marital status.

\subsubsection{Heart Patients Adherence-IMB Scale (HPA-IMBS)}

To date, a standardized scale has not been developed to measure the components of the IMB model for heartrelated patients' behaviors. An IMB measure used in a previous study on behavioral change was used in the present study [21] and guided the development of information, motivation and behavioral skills scale to measure heart-relevant behaviors among the participants. Face and content validity of this scale was assessed through peer review, and the internal consistency reliability for each measure was calculated through a pilot study of 60 heart patients. After several revisions, the final version of the scale was prepared. The Heart Patient Adherence IMB Scale consists of three subscales. The information subscale consisted of 22 multiple choice items which assessed one's information and knowledge on risk of heart disease and protective factors (e.g., Choose the correct answer: 1) High blood pressure is like common cold and has a short duration, 2) High blood pressure is reversed after a few months of using medication, 3) High blood pressure is a permanent condition and requires lifelong treatment). Maximum score possible for information subscale was 22, and Cronbach's alpha coefficient was 0.74 . Motivation subscale consisted of 25 items on a Likert scale with options ranging from not at all (0) to very much (5) assessed heart patients' attitudes and subjective norms for adherence (e.g., It frustrates me to think that I will have to take these medication every day for the rest of my life). Maximum score possible for this subscale was 125, and Cronbach's alpha coefficient was 0.89. Behavioral skills subscale consisted of 13 items on a Likert scale ranging from $0=$ never to $5=$ very often, assessed patients' behavioral skills and self-efficacy in regard to adherence (e.g., I can exercise regularly). Maximum score possible for this subscale was 65 , and Cronbach's alpha coefficient was 0.88 .

\subsubsection{General and Specific Adherence Scales}

The General and Specific Adherence Scales [22] were developed for assessing the adherence of patients with heart disease, diabetes and hypertension using a Likert scale with five items ranging from $1=$ Never to $6=$ All the time. The general measure of adherence assesses the patients' tendency to adhere to medical recommendations (e.g., I exactly followed my doctor's suggestions). The internal consistency reliability of the scale in current sample was acceptable (alpha $=0.47$ ). Specific adherence measures vary based on different medical condi- 
tions. The Specific Adherence measure used in the current study consisted of ten items assessing adherence to specific behaviors recommended for heart disease patients (e.g., stop or cut down on smoking). The internal consistency reliability of the scale in current sample was acceptable (alpha $=0.57)$.

\subsection{Procedure and Intervention}

The study employed a pre-posttest follow-up, experimental design to examine the effect of the brief IMB model-based intervention on patients' general and specific adherence. For pretest, participants were randomly assigned either to an intervention, or to a hospital standard care group using a randomization list. Participants in the intervention group participated in a pretest, theory-based intervention, posttest, and follow up, respectively. Those in the standard care control group received the pretest, posttest; follow up with no intervention beyond the standard care at the hospital for CABG patients. The patients in both groups completed pretest measures consisting of the Heart Patients Adherence-IMB Scale (HPA-IMBS), General Adherence Scale (GAS), and Specific Adherence Scale (SAS). Participants in the intervention group returned later in the day and took part in the IMB model-based intervention. Standard hospital care was offered to participants in both groups. Participants from both the intervention $(n=75)$ and standard-care control group $(n=77)$ returned one month after their pretest assessment to complete posttest measures and were reassessed three months later.

The IMB model of health behavior change was designed to address patients' deficits in regard to adherence information, motivation, and behavioral skills [16]. Intervention foci were derived from an extensive review of the literature, lengthy conversations with experts in the field, and elicitation work with patients in the study population. These inputs helped to tailor the intervention design, delivery, and content to the needs and characteristics of the CABG patients. Participants in the intervention group received one session (120 minutes) of IMB-based intervention in groups of five.

Information about heart disease risk factors and adherence behaviors (nutrition management, exercise, smoking cessation, cardiac rehabilitation, stress management, medication adherence, importance of adequate adherence to medical recommendations, costs of non-adherence and benefits of adherence) were presented using a variety of teaching aids, including showing a short educational film and providing handouts. The motivational component of the intervention was designed to help the patients identify, verbalize, and reinforce their positive attitudes and subjective normative support for adherent behaviors. The interventionist used motivational interview techniques such as providing personal feedback, asking open-ended questions, affirmations, reflective listening and negotiating goals that were realistic and feasible to enhance personal and social motivation to adherence to medical recommendations. The behavioral skills component focused on teaching how to effectively monitor nutrition, integrate physical activity into life style, quit smoking, control stress, and self-administer medications.

The patients randomly assigned to the control group received standard care at the hospital for CABG patients which included a combination of medical treatment, monitoring by physician, and group classes regarding risk factors for coronary heart disease, and self care behaviors after surgery.

\subsection{Data Analysis}

Descriptive statistics were calculated for all study variables. Chi Square $\left(\chi^{2}\right)$ was used to compare demographic characteristics. Path analysis was used to examine the relationships posited by the IMB model's constructs of CABG patients' information, motivation, behavioral skills and adherence using LISREL 8.53. Two separate models were created with 1) measures of general adherence behavior, and 2) measures of specific adherence behavior.

\section{Results}

The sample was predominantly male $(n=124,81.6 \%)$, married $(n=149,98 \%)$, of lower educational attainment (high school education or less, $n=121,79.6 \%$ ), and with mean age of 53.16 years ( $\mathrm{SD}=11.87$ ).

The relationship between IMB model constructs of information, motivation, behavioral skills, and adherence behaviors in CABG patients were measured using LISREL 8.53. Two structural models were examined that corresponded to the IMB model as initially described by Fisher and Fisher; one model for general adherence, and the other for specific adherence [17]. The likelihood ratio chi-square tests are reported for each model, but 
model fit was primarily evaluated with the goodness of fit index (GFI) and the root mean square error of approximation (RMSEA) [23]. The RMSEA is sensitive to over fit; that is, it begins to increase when too many paths have been included [24].

\subsection{IMB Model and General Adherence}

The IMB model predicts that adherence related information, motivation, and behavioral skills are determining factors of adherence behavior. To evaluate structural relations of constructs in the general adherence hypothesis, the direction and magnitude of the path coefficients were measured. The estimated model demonstrated good fit of the data (see Table 1). The proposed model with structural parameters and tests of significance of individual paths and indicators appears in Figure 1.

As can be seen in Figure 1, there were significant direct paths from motivation to behavioral skills and from motivation to general adherence. The association between information and behavioral skills was not significant. However, the association between information and motivation was significant. There was no significant association between behavioral skills and general adherence. In sum, consistent with predictions made by the IMB model of adherence, motivation was directly related to both general adherence behavior and behavioral skills. Relations between information and general adherence and also between behavioral skills and general adherence were mediated by motivation. The estimated model accounted for $63 \%$ of the variance in general adherence.

\subsection{IMB Model and Specific Adherence}

The IMB model predicts that behavior-specific information, motivation and behavioral skills are determining factors of adherence behavior. With regard to specific adherence, specific structural relations of the constructs in the model were evaluated through inspection of the direction and magnitude of the path coefficients. The estimated model demonstrated good fit of the data (see Table 2). The estimated model with structural parameters and tests of significance of individual paths and indicators appears in Figure 2.

Table 1. Estimated information-motivation-behavioral skills model of CABG patients' general adherence. Overall model fit. Fit indices: chi-square $\left(\chi^{2}>0.5\right)$, Root Mean Squared Error of Approximation (RMSEA $\left.<0.5\right)$, Normed-Fit Index (NFI > 0.9), Root Mean Square Residual (RMR < 0.5), Goodness of Fit Statistic (GFI > 0.9), Adjusted Goodness of Fit Statistic (AGFI > 0.9).

\begin{tabular}{ccc}
\hline Index & Values \\
\hline df & $\chi^{2}$ & $0.480 \mathrm{P}=0.489>0.5$ \\
$\chi^{2} / \mathrm{df}$ & $<2$ \\
RMSEA & $0.001<0.5$ & $0.997>0.9$ \\
NFI & $0.0130<0.5$ \\
GFI & $0.998>0.9$ \\
AGFI & $0.984>0.9$ \\
\hline
\end{tabular}

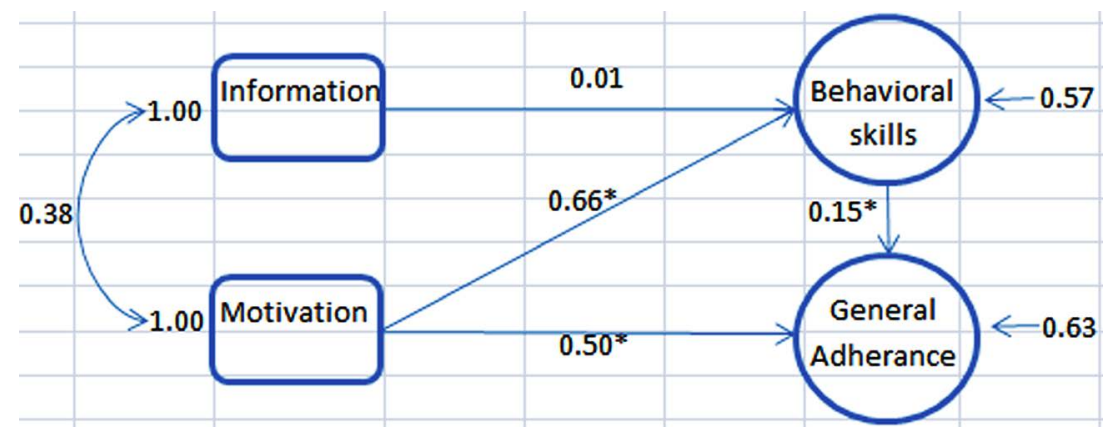

Figure 1. Estimation of the information-motivation-behavioral skills model of general adherence in CABG patients. Note: Coefficients are standardized path coefficients. For tests of significance of individual path, ${ }^{*} \rho<0.05,{ }^{* * *} \rho<0.01,{ }^{* * *} \rho<0.001$. 
Table 2. Estimated information-motivation-behavioral skills model of CABG patients' specific adherence. Overall model fit. Fit indices: chi-square $\left(\chi^{2}>0.5\right)$, Root Mean Squared Error of Approximation (RMSEA $\left.<0.5\right)$, Normed-Fit Index (NFI > 0.9), Root Mean Square Residual (RMR < 0.5), Goodness of Fit Statistic (GFI > 0.9), Adjusted Goodness of Fit Statistic (AGFI > 0.9).

\begin{tabular}{cc}
\hline Index & Values \\
\hline $\mathrm{df}$ & 1 \\
$\chi^{2}$ & $0.0376 \mathrm{P}=0.846>0.5$ \\
$\chi^{2} / \mathrm{df}$ & $<2$ \\
RMSEA & $0.0001<0.5$ \\
NFI & $1.00>0.9$ \\
RMR & $0.00355<0.5$ \\
GFI & $1.00>0.9$ \\
AGFI & $0.999>0.9$ \\
\hline
\end{tabular}

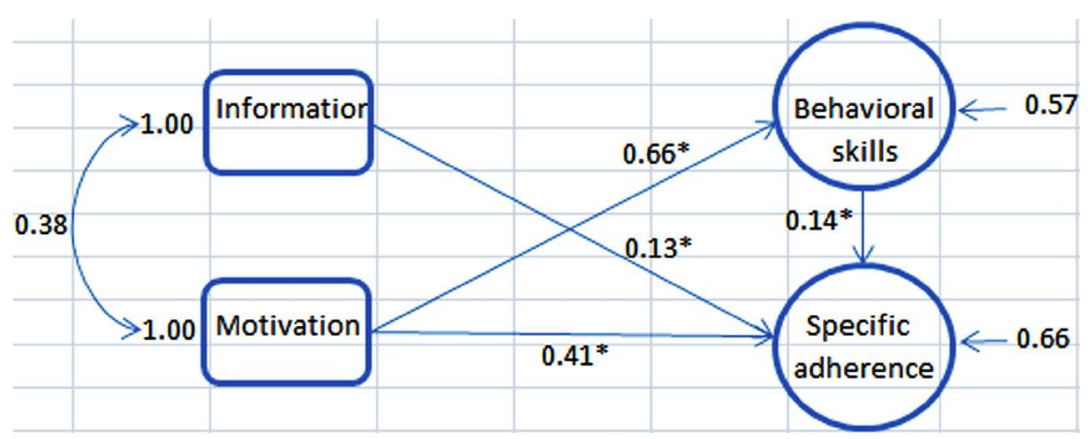

Figure 2. Estimation of the information-motivation-behavioral skills model of specific adherence in CABG patients. Note: Coefficients are standardized path coefficients. For tests of significance of individual path, ${ }^{*} \rho<0.05,{ }^{* *} \rho<0.01,{ }^{* * *} \rho<0.001$.

As predicted, there were significant paths from motivation to behavioral skills and from motivation to specific adherence. There was no direct relationship between information and specific adherence, and there was also no direct relationship between behavioral skills and specific adherence. Relations between information and specific adherence and also between behavioral skills and specific adherence were mediated by motivation. The full model accounted for $0.66 \%$ of the variance in specific adherence.

\section{Discussion}

The focus of this study was to evaluate the IMB model of adherence in CABG patients. This model of health behavior change [14] [21] was used in order to conceptualize the determining factors of adherence behavior in a sample of Iranian patients with CABG.

Consistent with the IMB model, having more information and motivation was associated with behavioral skills; and behavioral skills predicted adherence behavior. IMB model has suggested [21] that adherence-related information and motivation were each significantly associated with adherence-related behavioral skills, and that adherence related behavioral skills were significantly related to adherence behavior. Information and motivation could also bypass mediation through behavioral skills, and directly relate to adherence behavior.

Path analysis was used to test the extent to which the IMB model constructs explained the variability in adherence behaviors in the current study. These analyses also allowed for examining the relationship between IMB model constructs and possible modifications to the IMB model of adherence, and IMB model-based intervention in CABG patients. Overall, the IMB models of general and specific adherence fit the data. The IMB constructs accounted for a strong proportion of the variance in general adherence (63\%) and a somewhat larger proportion of the variance in specific adherence (66\%). Significant relationship among constructs indicated that motivation had direct relationship with general and specific adherence, and this was not mediated by behavioral skills. In addition, information and behavioral skills related significantly to motivation but not to adherence behavior. 
At this time, our results suggest that the motivation construct in the IMB model is well positioned to explain results in the sample data. Adherence related motivation in CABG patients was associated with information and behavioral skills, and was linked to adherence behavior. These findings build support for the utility of the IMB model in organizing core determinants of CABG patients' adherence, and suggest directions for IMB model based interventions, where motivation is directly targeted through intervention strategies.

The findings of this study indicate that motivation has a key role in improving adherence in Iranian CABG patients. This finding has been supported by observational studies with follow-up periods of up to 6 months among student and clinical populations. These studies have shown that affective attitude (the extent to which performing the behavior is enjoyable) is a stronger predictor of intention to perform or increase physical activity, than instrumental attitude (the extent to which performing the behavior is perceived to be beneficial) [25]-[27] Literature review results [28] indicated that motivational and self-management strategies could likely improve rehabilitation uptake and lifestyle change in patients with heart disease. In cross-sectional study [29], feelings associated with physical activity completely mediated the impact of attitude and social norms on physical activity, and partially mediated the impact of perceived control on behavior. Kalichman [30] reported that motivation impacted adherence behavior primarily through adherence-related behavioral skills, but no relation was found between adherence-related information and behavioral skills (which was originally predicted by the IMB model). Although other studies did not find motivation as a key factor; however, they showed that in model-based intervention with Puerto Ricans living with diabetes and HIV/AIDS, respectively information was directly related to adherence behavior, and behavioral skills were related to adherence behavior [20] [31].

The present results suggest that theory-based interventions could improve CABG patients' adherence when targeting improving motivation.

The IMB model-based intervention effectively improved general and specific adherence. Although perfect replication of the IMB model's predicted pathways was not achieved in this study, support for several of these pathways was demonstrated. Motivation was a significant predictor of adherence behavior, and was important to improve patient's acceptance and retention of desired behaviors. These findings suggest that interventions that are, patient-centered and interactive could produce more favorable results. These interventions could likely increase patients adherence-related motivation and, in turn, improve patient's understanding, acceptance and retention of adherence related knowledge and behavioral skills.

In keeping with the current trend towards theory-based heart patient education, this study provides empirical support for the Information-Motivation-Behavioral Skills (IMB) model of health behavior change as an appropriate, comprehensive health behavior change framework for CABG patient adherence behavior. The findings characterize the key element of CABG patient adherence and indicate that in addition to knowledge, and behavioral skills, CABG patients' education programs should mainly target motivation, and facilitate behavior change.

Our results also lend support to the growing body of literature and recommendations related to tailoring interventions to the motivational needs of patients [30]-[32]. Based on the findings of this study and consistent with the IMB model, providers and educators need to be cognizant of patients' level of motivation, and tailor their educational programs accordingly. Such interventions are likely to be more effective at producing behavior change than ad-hoc, knowledge-based programs alone.

Prior to conducting this study, standardized measures of adherence information, motivation, behavioral skills in patients with heart disease had not been developed. Thus, the reliability and validity of these measures are limited to the findings of this study. A common limitation of the current research is that the data on adherence behaviors were self-reported, as in most research studies on adherence. Our results speak most clearly to the population under study, and should be replicated in different patient groups. The strengths of this research include using a theoretical model to design an intervention, and to evaluate a cost effective, feasible intervention that could be integrated into general routine patient care. The findings from this study provide suggestions for designing and implementing health behavior change interventions that take into consideration specific populations and their needs. Additional research is needed to confirm the obtained pattern of associations for adherence-related IMB constructs in CABG patients. Investigation longitudinal effects of IMB model constructs on changes in adherence behavior in patients with cardiovascular disease and application of the model to other pressing health-related issues (e.g., understanding adoption and maintenance of a broad array of health behavior changes, understanding and increasing use of other prevention behaviors, understanding) might be useful avenues to pursue. 


\section{References}

[1] Charlson, M.E. and Isom, O.W. (2003) Clinical Practice: Care after Coronary-Artery Bypass Surgery. The New England Journal of Medicine, 348, 1456-1463. http://dx.doi.org/10.1056/NEJMcp010691

[2] Karthik, S., Tahir, N., Thakur, B. and Nair, U. (2006) Risk Factor Awareness and Secondary Prevention of Coronary Artery Disease: Are We Doing Enough? Interactive Cardiovascular and Thoracic Surgery, 5, 268-271. http://dx.doi.org/10.1510/icvts.2005.120956

[3] Mc Call, L.A. and Martin Ginis, K.A. (2004) The Effects of Message Framing on Exercise Adherence and Health Beliefs among Patients in a Cardiac Rehabilitation Program. Journal of Applied Biobehavioral Research, 9, 22-135.

[4] Ben-Zur, H., Rappaport, B., Ammar, R. and Uretzkky, G. (2000) Coping Strategies, Life Style Changes, and Pessimism after Open-Heart Surgery. Health \& Social Work, 25, 201-209. http://dx.doi.org/10.1093/hsw/25.3.201

[5] Leong, J., Molassiotis, A. and Marsh, H. (2004) Adherence to Health Recommendation after a Cardiac Rehabilitation Program in Post-Myocardial Infarction Patients: The Role of Health Beliefs, Locus of Control and Psychological Status. Clinical Effectiveness Nursing, 8, 26-38. http://dx.doi.org/10.1016/j.cein.2004.02.001

[6] Lynam, I., Catl, D., Goggin, K., Rabinowits, J.L., Gerkovich, M.M., Williams, K. and Wright, J. (2009) Autonomous Regulation and Locus of Control as Predictors of Antiretroviral Medication Adherence. Journal of Humanistic Psychology, 14, 578-586.

[7] J. Woodgate and Brawley, L.R. (2008) Self-Efficacy for Exercise in Cardiac Rehabilitation: Review and Recommendations. Journal of Health Psychology, 13, 366-387. http://dx.doi.org/10.1016/j.cein.2004.02.001

[8] Alm-Roije, C., Stagmo, M., Udén, G. and Erhardt, L. (2004) Better Knowledge Improves Adherence to Lifestyle Changes and Medication in Patients with Coronary Heart Disease. European Journal of Cardiovascular Nursing, 3, 321-330. http://dx.doi.org/10.1016/j.ejcnurse.2004.05.002

[9] Fonarow, G.C., Gawlinski, A., Moughrabi, S. and Tillisch, J.H. (2001) Improved Treatment of Coronary Heart Disease by Implementation of a Cardiac Hospitalization Atherosclerosis Management Program (CHAMP). American Journal of Cardiology, 87, 819-822. http://dx.doi.org/10.1016/S0002-9149(00)01519-8

[10] Fonarow, G.C. and Ballantyne, C.M. (2001) In-Hospital Initiation of Lipid Lowering Therapy for Patients with Coronary Heart Disease: The Time Is Now. Circulation, 103, 2768-2770. http://dx.doi.org/10.1161/01.CIR.103.23.2768

[11] Bandura, A. (1997) Self-Efficacy: The Exercise of Control. W. H. Freeman and Company, New York, 25-30.

[12] Hardeman, W., Kinmonth, A.L., Michie, S. and Sutton, S. (2011) Theory of Planned Behaviour Cognitions Do Not Predict Self-Reported or Objective Physical Activity Levels or Change in the ProActive Trial. British Journal of Health Psychology, 16, 135-150. http://dx.doi.org/10.1348/135910710X523481

[13] Mihalko, S.L., Brenes, G.A., Farmer, D.F., Katula, J.A., Balkrishnan, R. and Bowen, D.J. (2004) Challenges and Innovations in Enhancing Adherence. Controlled Clinical Trials, 33, 775-790.

[14] Fisher, J.D. and Fisher, W.A. (2002) The Information-Motivation Behavioral Skills Model. In: Diclemente, R.J., Crosby, R.A. and Kegler, M.C., Eds., Emerging Theories in Health Promotion Practice and Research: Strategies for Improving Public Health, Jossey-Bass, Inc., Indianapolis.

[15] Fisher, J.D. and Fisher, W.A. (2000) Theoretical Approaches to Individual-Level-Change in HIV Risk Behavior. In: Peterson, J.I. and DiClemente, R.J., Eds., Handbook of HIV Prevention, Klumer Academic/Plenum Publishers, New York, 3-55.

[16] Fishe, W.A., Fisher, J.D. and Harman, J.J. (2003) The Information-Motivation-Behavioral Skills Model: A General Social Psychological Approach to Understanding and Promoting Health Behavior. In: Suls, J. and Wallston, K., Eds., Social Psychological Foundations of Health and Illness, Blackwell, Malden, 82-106. http://dx.doi.org/10.1002/9780470753552.ch4

[17] Fisher, J.D. and Fisher, W.A. (1992) Changing AIDS-Risk Behavior. Psychological Bulletin, 111, 455-474. http://dx.doi.org/10.1037/0033-2909.111.3.455

[18] Zarani, F., Besharat, M.A., Sadeghian, S. and Sarami, G. (2010) The Effectiveness of the Information-Motivation-Behavioral Skills Model in Promoting Adherence in CABG Patients. Journal of Health Psychology, 15, 828-837. http://dx.doi.org/10.1177/1359105309357092

[19] Zarani, F., Besharat, M.A., Sarami, G. and Sadeghian, S. (2012) An Information-Motivation-Behavioral Skills (IMB) Model-Based Intervention for CABG Patients. International Journal of Behavioral Medicine, 19, 543-549. http://dx.doi.org/10.1007/s12529-011-9193-2

[20] Starace, F., Massa, A., Amico, K.R. and Fisher, J.D. (2006) Adherence to Antiretroviral Therapy: An Imperical Test of the Information-Motivation-Behavioral Skills Model. Health Psychology, 25, 153-162.

http://dx.doi.org/10.1037/0278-6133.25.2.153 
[21] Fisher, J.D., Fisher, W.A., Amico, K.R. and Harman, J.J. (2004) An Information-Motivation-Behavioral Skill Model of Adherence to Antiretroviral Therapy. Health Psychology, 25, 462-475. http://dx.doi.org/10.1037/0278-6133.25.4.462

[22] Hays, R.D., Kravitz, R.L., Mazel, R.M., Sherbourne, C.D., Dimatteo, M.R., Rogers, W.H. and Greenfield, S. (1994) The Impact of Patient Adherence on Health Outcomes for Patients with Chronic Disease in the Medical Outcome Study. Journal of Behavioral Medicine, 17, 347-360. http://dx.doi.org/10.1007/BF01858007

[23] Brown, M.W. and Cudeck, R.I. (1993) Alternative Ways of Assessing Model Fit. In: Bollen, K. and Long, K., Eds., Testing Structural Equation Models, Sage, Newbury Park, 136-162.

[24] Ridgon, E. (1996) CFI versus RMSEA: Comparison of Two Fit Indexes for Structural Equation Modeling. Structural Equation Modeling, 3, 369-379. http://dx.doi.org/10.1080/10705519609540052

[25] French, D.P., Sutton, S., Hennings, S.J., Mitchell, J., Wareham, N.J., Griffin, S. and Kinmonth, A.L. (2005) The Importance of Affective Beliefs and Attitudes in the Theory of Planned Behavior: Predicting Intentions to Increase Physical Activity. Journal of Applied Social Psychology, 35, 1824-1848. http://dx.doi.org/10.1111/j.1559-1816.2005.tb02197.x

[26] Karvinen, K.H., Courneya, K.S., Campbell, K.L., Hennings, S.J., Mitchell, J., Wareham, N.J., Griffin, S. and Kinmonth, A.L. (2007) Correlates of Exercise Motivation and Behavior in a Population-Based Sample of Endometrial Cancer Survivors: An Application of the Theory of Planned Behavior. International Journal of Behavioral Nutrition and Physical Activity, 4, 21-25. http://dx.doi.org/10.1186/1479-5868-4-21

[27] Rhodes, R.E., Blanchard, C.M., Hunt Matheson, D. and Coble, J. (2006) Disentangling Motivation, Intention, and Planning in the Physical Activity Domain. Psychology of Sport and Exercise, 7, 15-27. http://dx.doi.org/10.1016/j.psychsport.2005.08.011

[28] Beswick, A.D., Rees, K., West, R.R., Taylor, F.C. and Burke, M. (2005) Improving Uptake and Adherence in Cardiac Rehabilitation: Literature Review. Advanced Nursing, 49, 538-555. http://dx.doi.org/10.1111/j.1365-2648.2004.03327.x

[29] Kiviniemi, M.T., Voss-Hunke, A.M. and Seifert, A.L. (2007) How Do I Feel about the Behavior? The Interplay of Affective Associations with Behaviors and Cognitive Beliefs as Influences on Physical Activity Behavior. Health Psychology, 26, 152-158. http://dx.doi.org/10.1037/0278-6133.26.2.152

[30] Kalichman, S.C., Rompa, D., DiFonzo, K., Simpson, D., Austin, J. and Jacobson, L.P. (2001) HIV Treatment Adherence in Women Living with HIV/AIDS: Research Based on the Information-Motivation-Behavioral Skills Model of Health Behavior. Journal of the Association of Nurses in AIDS Care, 12, 58-67. http://dx.doi.org/10.1016/S1055-3290(06)60217-3

[31] Osborn, C.Y. and Fisher, J.D. (2008) Diabetes Education: Integrating Theory, Cultural Considerations, and Individually Tailored Content. Clinical Diabetes, 26, 148-150. http://dx.doi.org/10.2337/diaclin.26.4.148

[32] Osborn, C.Y., Rivet Amico, K. and Fisher, W.A. (2010) An Information-Motivation-Behavioral Skills Analysis of Diet and Exercise Behavior in Puerto Ricans with Diabetes. Journal of Health Psychology, 15, 1201-1213. http://dx.doi.org/10.1177/1359105310364173 